\title{
Enantioselective Epoxidation of Alkenes
}

\section{Catalyzed by 2-Fluoro- $N$-Carbethoxytropinone and related Tropinone Derivatives}

Alan Armstrong*, Ghafoor Ahmed, Belen Dominguez-Fernandez, Barry R. Hayter, and J.

Steven Wailes

\section{Supporting Information}

\section{Contents}

General Experimental Details

$\mathrm{S} 2$

Preparation of alkenes for epoxidation

$\mathrm{S} 2$

Characterisation Data for Product Epoxides in Table 2

S2

Determination of epoxide enantiomeric purity in Table 2

ORTEP representation of X-ray crystallographic data for fluoroketone $\mathbf{2}$

${ }^{1} \mathrm{H}$ NMR spectrum of fluoroketone 2

${ }^{1} \mathrm{H}$ NMR spectrum of alcohol 6

${ }^{1} \mathrm{H}$ NMR spectrum of enone 9

S8

${ }^{1} \mathrm{H}$ NMR spectrum of acetonide $\mathbf{1 0}$

S9 


\section{General experimental details}

Diethyl ether and tetrahydrofuran solvents were distilled from sodium-benzophenone ketyl; toluene from sodium; and dichloromethane from calcium hydride. Petrol refers to petroleum ether b.p. $40-60^{\circ} \mathrm{C}$ which was distilled prior to use. Other solvents and reagents were purified by standard procedures as necessary. Flash chromatography was performed on Merck Kieselgel 60 (230-400 mesh) unless otherwise stated. Analytical thin layer chromatography was performed using pre-coated glass-backed plates (Merck Kieselgel $60 \mathrm{~F}_{254}$ ). Chemical shifts for ${ }^{1} \mathrm{H}$ and ${ }^{13} \mathrm{C}$ NMR are expressed in ppm relative to internal $\mathrm{CHCl}_{3}, 7.26 \mathrm{ppm}$ ) or $\mathrm{CDCl}_{3}(77.0 \mathrm{ppm}, \mathrm{t})$. J values are measured in Hertz. Multiplicities

in ${ }^{13} \mathrm{C}$ spectra were determined by DEPT experiments. IR spectra were recorded as thin films on $\mathrm{NaCl}$ plates unless otherwise stated.

\section{Preparation of alkenes for epoxidation}

The following alkene substrates in Table 2 were not commercially available and were synthesised according to literature methods:

1-t-Butyldimethylsiloxy-1-phenylpropene (entry 18): Adam, W.; Fell, R.T.; Stegmann, V.R.; Saha-Moller, R.C. J. Am. Chem. Soc. 1998, 120, 708.

Benzoic acid cyclohex-1-enyl ester (entries 19, 20): Al Adel, I.; Salami, B.A.; Lavisalles, J.; Rudler, H. Bull. Soc. Chim. Fr. 1976, 903.

\section{Characterisation Data for Product Epoxides in Table 2}

The epoxide products are all literature compounds:

Entries 1-2 (trans-stilbene oxide): Imuta, M.; Ziffer, H.J. J. Org. Chem. 1979, 44, 25052509. Chang, H.-T.; Sharpless, K.B. J. Org. Chem. 1996, 61, 6456-6457.

Entry 3 (trans- -methylstyrene oxide): Witkop, B.; Foltz, C.M. J. Am. Chem. Soc. 1957, 79, 197-210.

Entry 4 (trans- -(hydroxymethyl)styrene oxide): Melloni, P.; Della, T.A.; Lazzari, E.; Mazzini, G.; Meroni, M. Tetrahedron 1985, 41, 1393.

Entry 5: Gao, Y.; Hanson, R.M.; Klunder, J.M.; Ko, S.Y.; Masamune, H.; Sharpless, K.B. J. Am. Chem. Soc. 1987, 109, 5765-5780. 
Entry 6: Garcia Martinez, A.; Oliver Ruiz, M.; Contelles, J. L. M. Synthesis 1986, 125128. Sugita, K.; Ohta, A.; Onaka, M.; Izumi, Y. Chem.Lett. 1990, 481-484.

Entry 7 (trans-5-decene oxide): Tanner, D.; Birgersson, C.; Gogoll, A.; Luthman, K. Tetrahedron 1994, 50, 9797-9824.

Entries 8-11 (phenylstilbene oxide and 1-phenylcyclohexene oxide): Brandes, B.D.; Jacobsen, E.N. J. Org. Chem. 1994, 59, 4378-4380.

Entry 12 (3,4-dihydronaphthalene oxide): Akhtar, M.N.; Boyd, D.R.; Hamilton, J.G. J. Chem. Soc., Perkin Trans. 1 1979, 2437-2440.

Entry 13 ( -methylstyrene oxide): Fujisawa, T.; Takemura, I.; Ukaji, Y. Tetrahedron Lett. 1990, 31, 5479-5482. Archelas, A.; Furstoss, R. J. Org. Chem. 1999, 64, 6112.

Entry 14-15 (styrene oxide): Robinson, P. L.; Barry, C. N.; Kelly, J. W.; Evans, S. A. J. Am. Chem. Soc. 1985, 107, 5210-5219.

Entry 16: (trans-ethyl cinnamate oxide): Adolfsson, H.; Coperet, C.; Chiang, J. P.; Yudin, A. K. J. Org. Chem. 2000, 65, 8651-8658.

Entry 17: (E-chalcone oxide) Augustyn, J. A. N.; Bezuidenhoudt, B. C. B.; Ferreira, D. Tetrahedron 1990, 46, 2651-2660.

Entry 18 ( -hydroxypropiophenone): Demir, A. S.; Hamamci, H.; Sesenoglu, O.; Aydogan, F.; Capanoglu, D.; Neslihanoglu, R.. Tetrahedron: Asymmetry 2001, 12, 19531956.

Entries 19-20 (1-benzyloxycyclohexene oxide): Zhu, Y.; Shu, L.; Tu, Y.; Shi, Y. J. Org. Chem. 2001, 66, 1818-1826. 


\section{Determination of epoxide enantiomeric purity in Table 2}

Entries 1 and 2: chiral HPLC on Chiracel OD, $10 \% i \mathrm{PrOH} /$ hexane, $0.8 \mathrm{ml} / \mathrm{min}$, detecting at $254 \mathrm{~nm}$, as detailed by Shi: Tu, Y; Wang, Z-X.; Shi, Y. J. Am. Chem. Soc., 1996, 118, 9806. Absolute configuration determined by comparison to Shi's results.

Entries 3-6, 8-12, 14, 15: ee and configuration determined by ${ }^{1} \mathrm{H}$ NMR in the presence of $\mathrm{Eu}(\mathrm{hfc})_{3}$ as chiral shift reagent, as detailed by Shi: Tu, Y; Wang, Z-X.; Shi, Y. J. Am. Chem. Soc., 1996, 118, 9806. Absolute configuration determined by comparison to Shi's results.

Entry 7: ee determined by ring opening with $\mathrm{NaOMe} / \mathrm{MeOH}$ followed by formation of the acetate and ${ }^{1} \mathrm{H}$ NMR analysis in the presence of $\mathrm{Eu}(\mathrm{hfc})_{3}$ as chiral shift reagent, as detailed by Shi: Tu, Y; Wang, Z-X.; Shi, Y. J. Am. Chem. Soc., 1996, 118, 9806. Absolute configuration determined by comparison to Shi's results.

Entry 13: chiral HPLC on Chiracel OD, 5\% $i \mathrm{PrOH} /$ hexane, $0.8 \mathrm{ml} / \mathrm{min}$, detecting at 254 nm, as detailed by Shi: Wang, Z. X.; Tu, Y.; Frohn, M.; Zhang, J. R.; Shi, Y. J. Am. Chem. Soc. 1997, 119, 11224-11235. Absolute configuration determined by comparison to Shi's results.

Entry 16: chiral HPLC on Chiracel OJ, 10\% $i \mathrm{PrOH} /$ hexane, $1.0 \mathrm{ml} / \mathrm{min}$, detecting at 254 $\mathrm{nm}$, retention times $6.99 \mathrm{~min}$ (minor enantiomer), $10.74 \mathrm{~min}$ (major enantiomer). Major enantiomer assigned as $(2 R, 3 S)$ by comparison of the sign of the product's optical rotation to the literature value of []$_{\mathrm{D}}+152$ for the $(2 S, 3 R)$ isomer: Cabon, O.; Larchevêque, M.; Buisson, D.; Azerad, R. Tetrahedron Lett. 1992, 38, 7337-7340.

Entry 17: chiral HPLC on Chiracel OD, 5\% EtOH/hexane, $1.0 \mathrm{ml} / \mathrm{min}$, detecting at 254 $\mathrm{nm}$, retention times $9.45 \mathrm{~min}$ (minor enantiomer), $10.87 \mathrm{~min}$ (major enantiomer).

Entry 18: chiral HPLC on Chiracel OD: Adam, W.; Diaz, M.T.; Fell, R. T.; Saha-Moller, C. R.. Tetrahedron: Asymmetry 1996, 7, 2207-2210.

Entries 19-20: chiral HPLC on Chiracel OD, as detailed by Shi: Zhu, Y.; Shu, L.; Tu, Y.; Shi, Y. J. Org. Chem. 2001, 66, 1818-1826. 
ORTEP representation of X-ray crystallographic data for fluoroketone 2 CIF file is also included separately as Supporting Information.

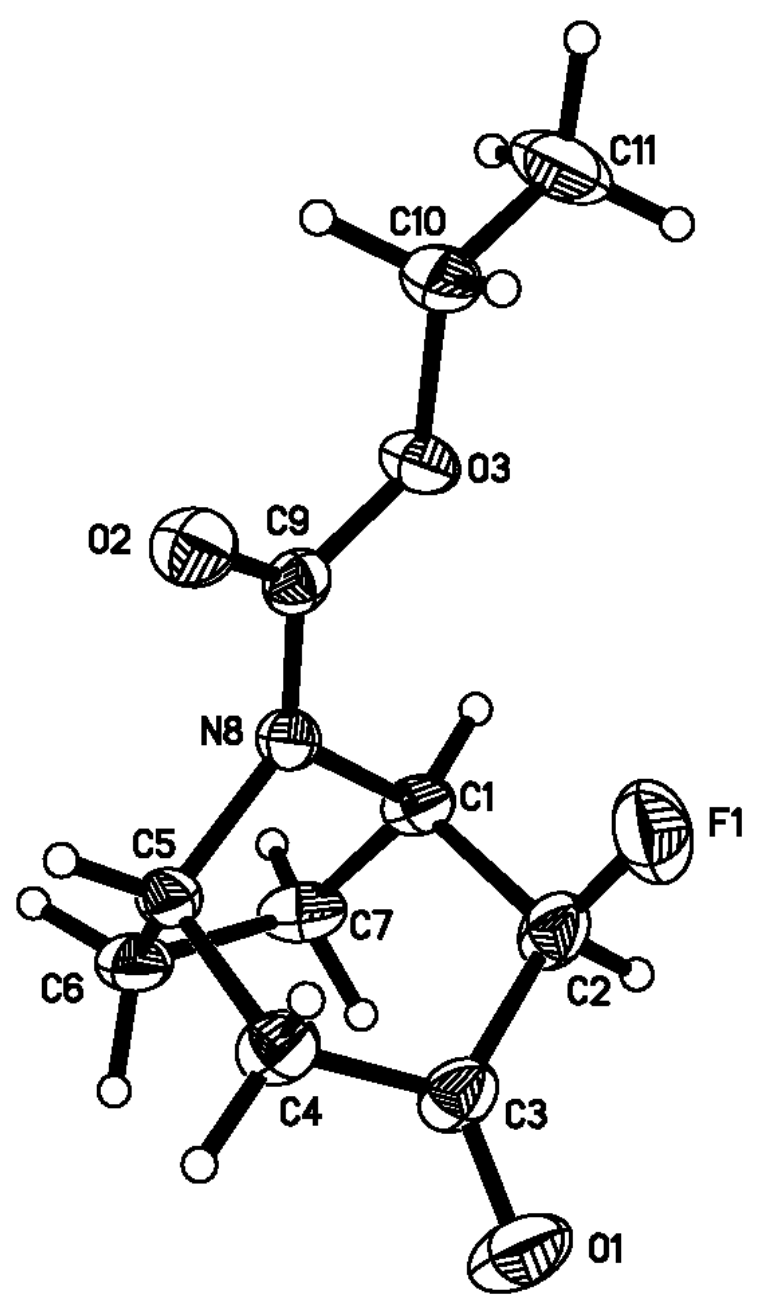




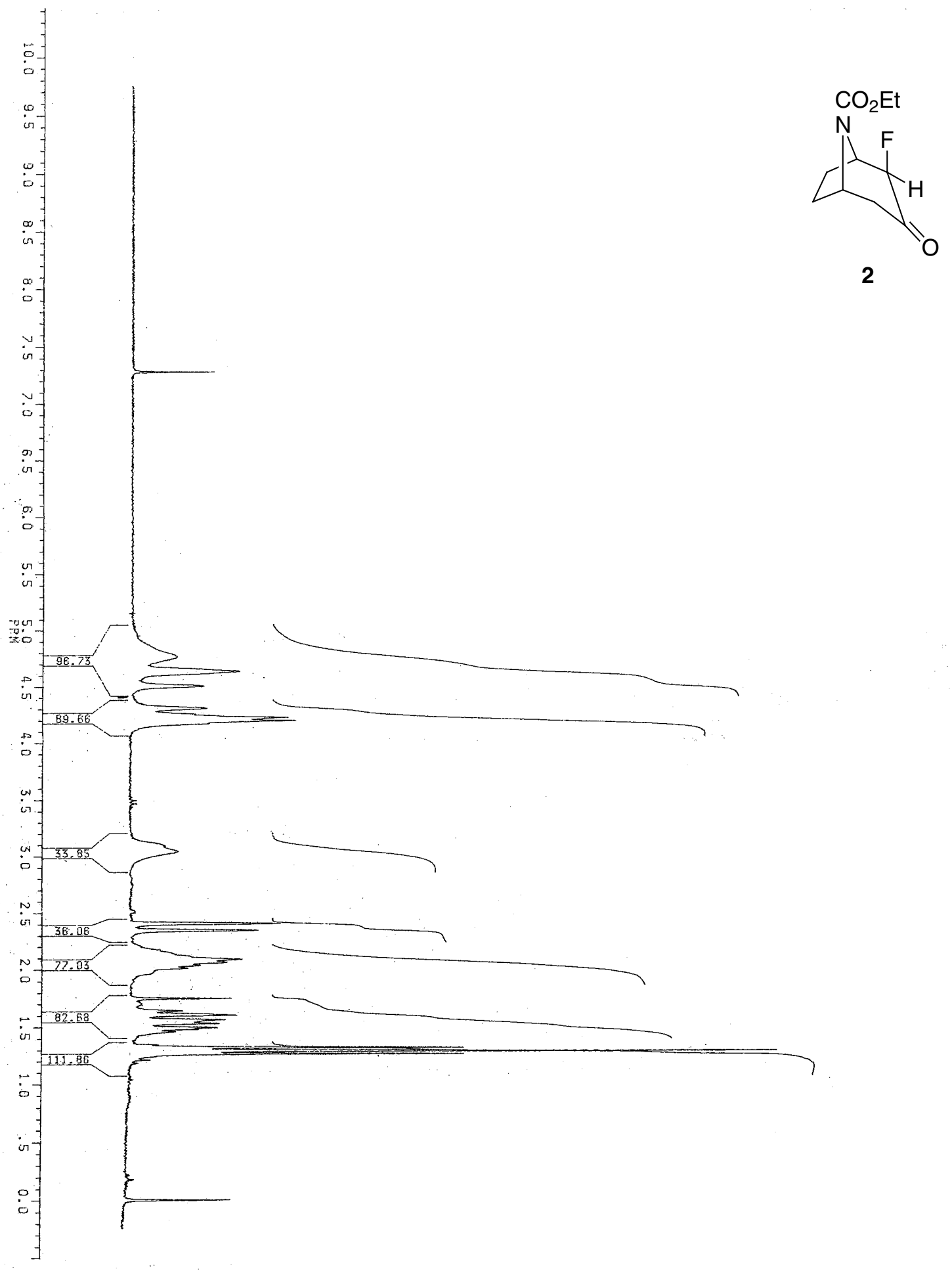



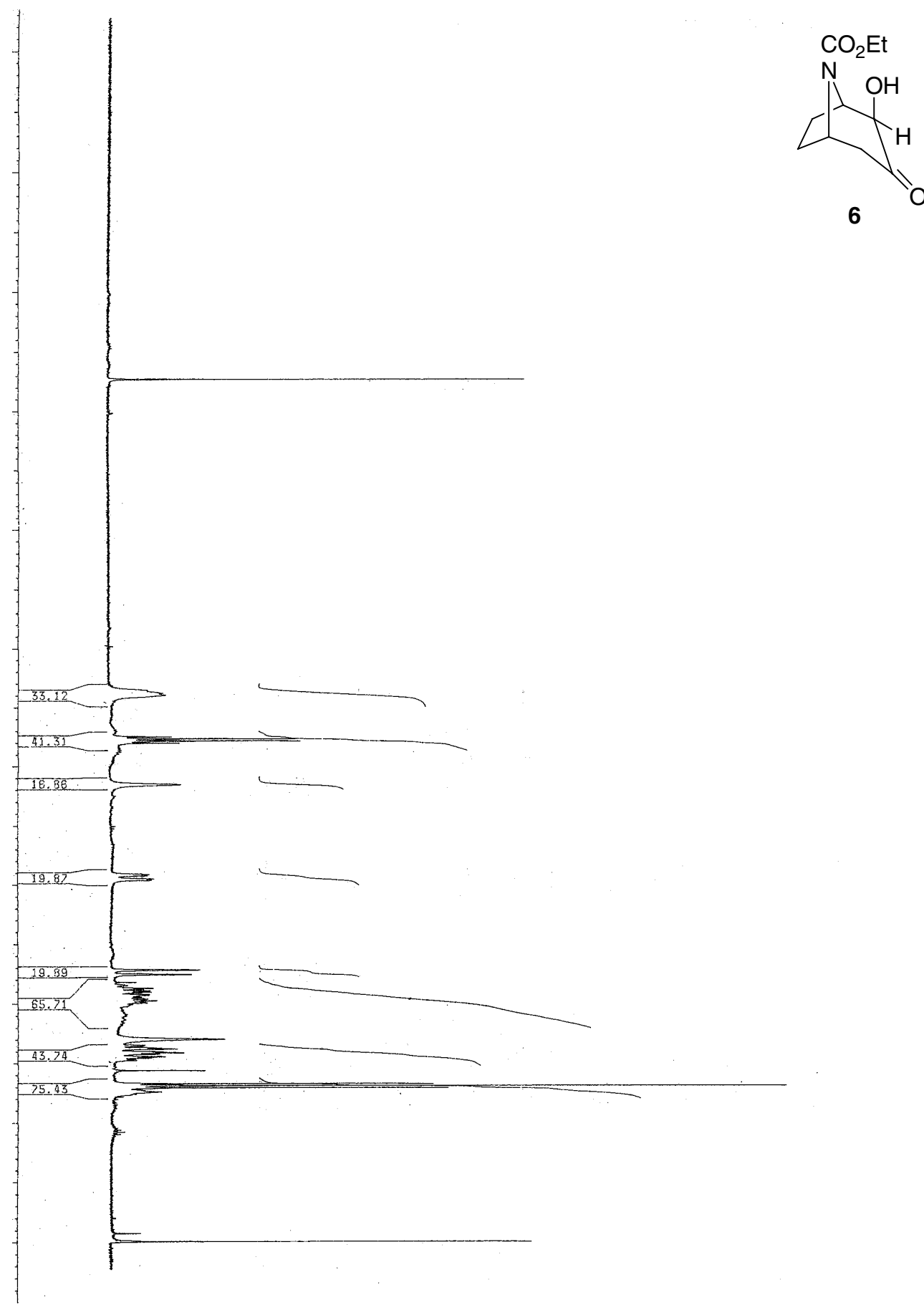


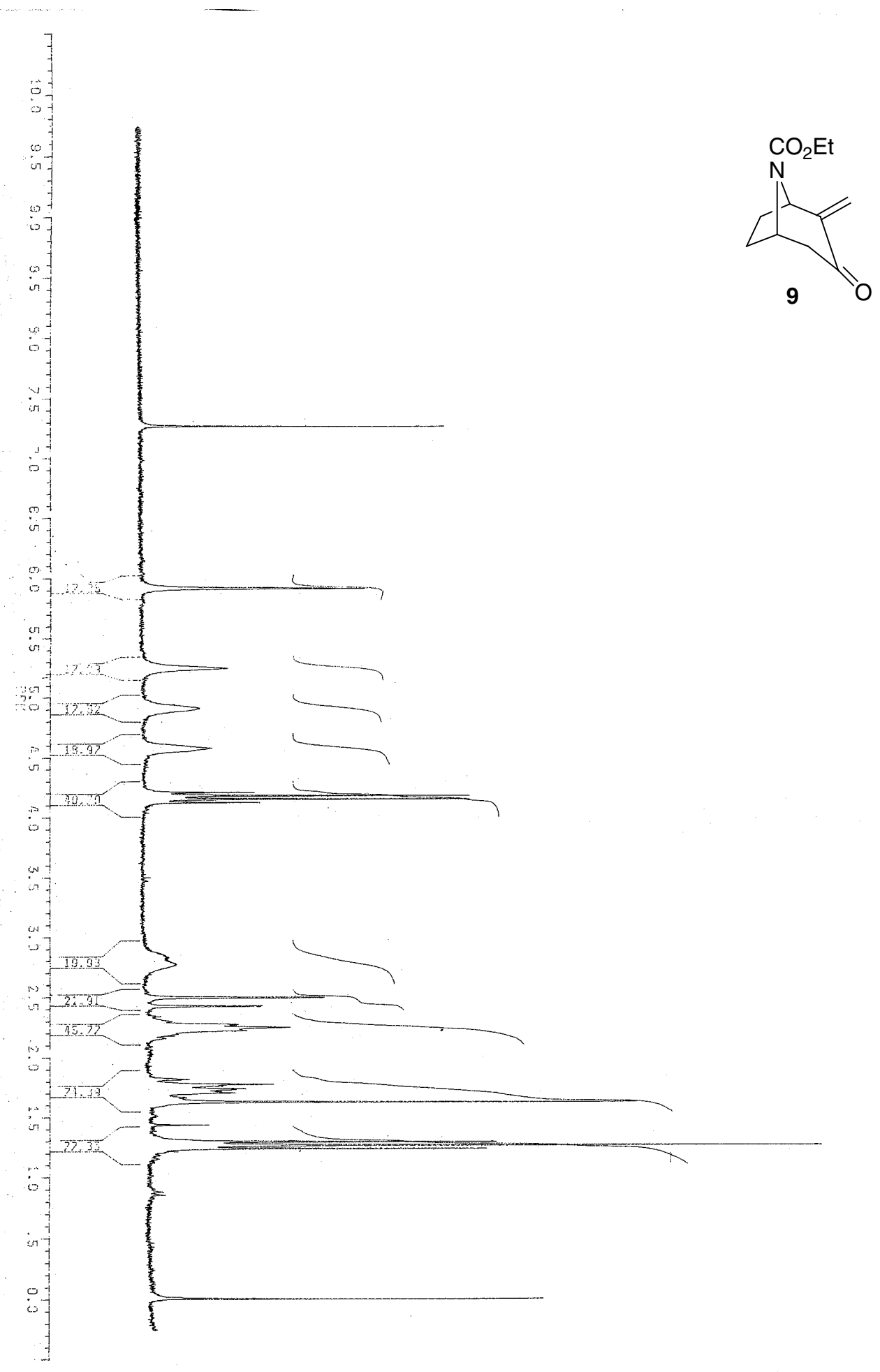




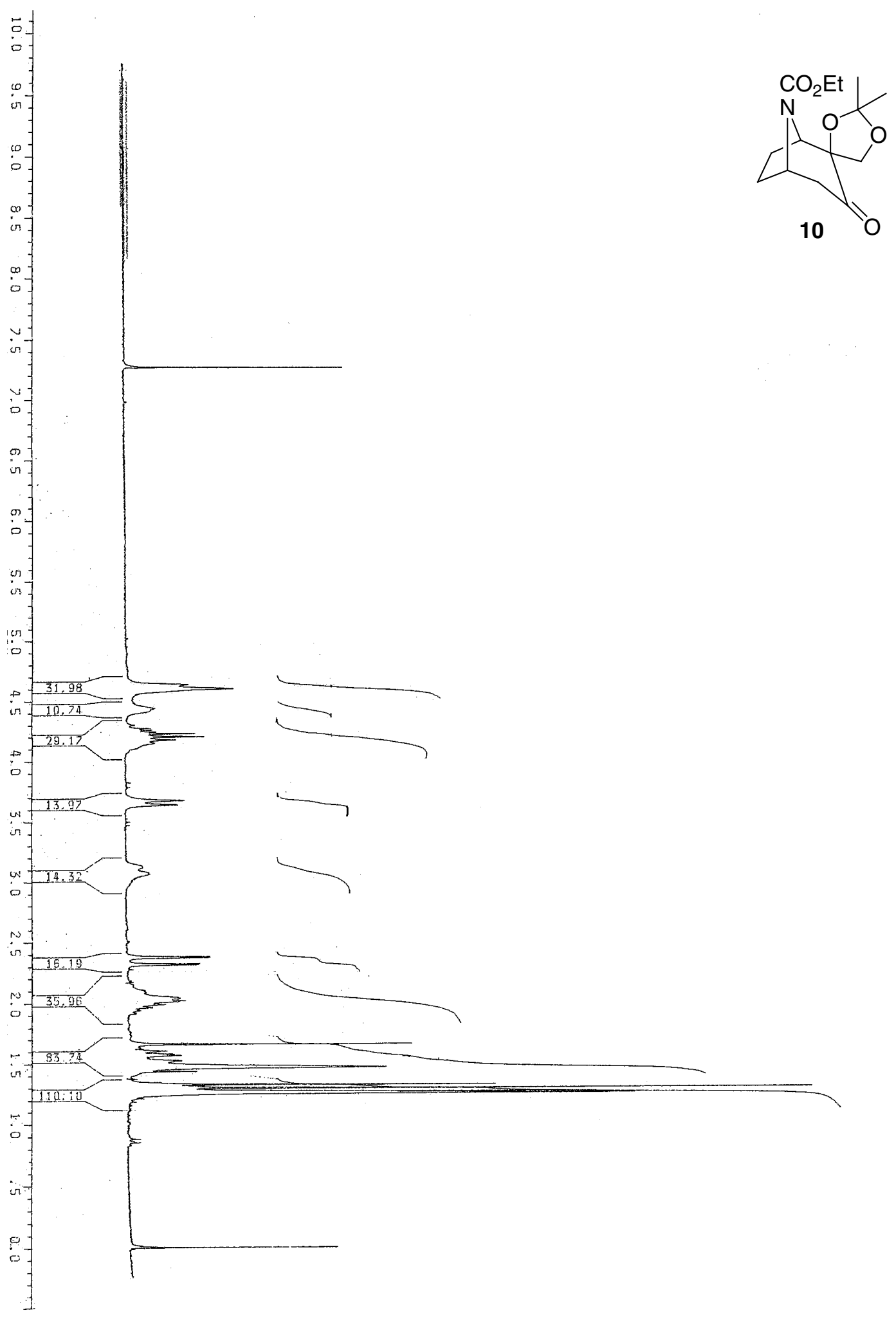

